\title{
ON THE DETERMINATION OF OPTIMAL TIME HORIZON IN A CONTROL PROBLEM IN NATURAL RESOURCE ECONOMICS
}

\author{
RAMESH C. KUMAR \\ and \\ FADLE M. NAQIB \\ Department of Economics \\ University of Waterloo \\ Waterloo, Ontario, N2L 3G1 \\ CANADA
}

(Received August 20, 1993 and in revised form December 18, 1993)

ABSTRACT. This paper is concerned with issues relating to the determination of optimal time horizon in a typical control problem of optimal extraction of an exhaustible natural resource. The paper extends the recent Highfill-McAsey results to cover all strictly concave utility functions.

KEY WORDS AND PHRASES. Optimal control, exhaustible resources, time horizon. 1991 AMS SUBJECT CLASSIFICATION CODE. 49K15, 90A16

\section{INTRODUCTION.}

In a recent article in this Journal, Highfill and McAsey [1] study the following optimal control problem which they describe as "the first problem in the economics of exhaustible natural resources."

subject to

$$
\underset{\langle q(t)\rangle}{\operatorname{Maximize}} J=\int_{0}^{T} U(q(t)) e^{-\delta t} d t
$$

$$
\begin{array}{ll}
\dot{x}(t)=-q(t), & q(t) \geq 0 \\
x(0)=x_{0} & \text { given } \\
x(T) \geq 0 & \text { free } \\
T \geq 0 & \text { free }
\end{array}
$$

where $x(t)$ is the remaining (in ground) stock of an exhaustible, natural resource at time $t ; q(t)$ is the instantaneous extraction rate, $T$ the time (planning) horizon and $U($.$) a non-negative, strictly concave$ utility function which is discounted over time at rate $\delta>0$.

The authors prove two distinct results. While the main thrust of their analysis is directed toward demonstrating that the strict concavity of the utility function alone may not be sufficient for ensuring the existence of an optimal solution for arbitrarily chosen $0 \leq x_{0} \leq \infty$, and that the additional requirement of "asymptotic non-linearity" will suffice (Example 2.3 and Theorem 3.1), it is their other result (Theorem 2.2) which is perhaps of greater significance for economists. Specifically, this result is concerned with the determination of the optimal time horizon and purports to prove that optimal $T<\infty$ if and only if $\lim _{q \rightarrow 0} U^{\prime}(q)<\infty$. The proposition that an elementary property of the utility function is sufficient for determining whether the optimal planning horizon ought to be finite or infinite, without ever invoking economic concepts of impatience and time-discounting of utility, is certainly not well established in the literature even though the problem has been considered before by Vousden [2] in a different context. 
Highfill and McAsey have clearly made an important and useful contribution Their analysis however is confined to the special case of strictly concave, non-negative utility function that also satisfies the requirement that $U(0)=0$ This restriction is certainly appropriate if the control problem above is viewed strictly as a "cake-eating problem" - and this is not uncommon in the literature - with the obvious stipulation that no cake implies no utility Given that economic theory demands no such restrictions. it precludes two situations where the issue of the determination of optimal planning horizon can be fruitfully investigated the case where the utility function may assume negative values over a range and the situation where $U(0)$ may not be zero Such situations can arise even in the context of the "first control problem" if either we allow for the possibility that utility is derived not from the direct consumption of the resource stock (the "cake") but from that of another good in whose production the exhaustible resource is an input, or consider the case where an account is taken of utility arising from the fixed consumption of one or more other commodities The utility functions $U(q)=L n(q) ; q>0$ and $U(q)=a+(q+b)^{\eta}$, $a>0, b>0,1>\eta>0$, both extensively used in economics, illustrate these possibilities The purpose of this paper is to extend the Highfill-McAsey analysis to cover all strictly concave utility functions.

In the next section, we begin by arguing that the size of the terminal extraction rate and the corresponding value of the utility function are both crucial in determining the duration of the planning horizon. We then present three results that, in addition to confirming Highfill-McAsey result, extend the analysis as mentioned above

\section{THE OPTIMAL TIME HORIZON.}

The necessary condition for the maximization of the Hamiltonian function, $H($, ).

$$
H\left(t ; x, q, \lambda=e^{-\delta t} U(q(t))-\lambda(t) q(t)\right.
$$

of the control problem of Section 1 is

$$
\begin{array}{rlrl}
e^{-\delta t} U^{\prime}(q(t)) & =\lambda(t) ; & & q(t)>0 \\
<\lambda(t) ; & & q(t)=0
\end{array}
$$

where $\lambda(t)$ is the co-state variable associated with the state $x(t)$. In addition to $(22)$ and the state equation $\dot{x}(t)=-q(t)$, the necessary conditions for solving the control problem include

$$
\dot{\lambda}(t)=-\frac{\delta H}{\delta x}=0
$$

which in conjunction with (2.2) implies that the optimal $\lambda(t)$ is a positive constant, say $\lambda$, and that $q(t)$ declines continuously with time, and the transversality condition:

$$
\left[H-\lambda \phi^{\prime}\right]_{t=: T}=0
$$

where

$$
\phi(T)=X_{0}-\int_{0}^{T} q(t) d t
$$

Chiang [3] may be consulted for the derivation of the condition. In the context our problem (2.4) implies

$$
e^{-\delta T} U(q(T))=0
$$

which may be used to infer about the magnitude of optimal $q(T)$. It is obvious that if optimal $T<\infty$, the above condition can be satisfied only if $U(q(T))=0$. On the other hand, if optimal $T$ is infinite, (2.5) will be satisfied whenever $|U(q(T))|<\infty$. Clearly then the values of both the optimal $q(T)$ and $U(q(T))$ are crucial for whether optimal $T$ is finite or not. These are in turn determined by the type of the utility function one considers. All strictly concave utility functions belong to one of the three types 
depicted in the following diagram Highfill and McAsey have considered Type I functions The two examples of Section 1 refer respectively to Types II and III

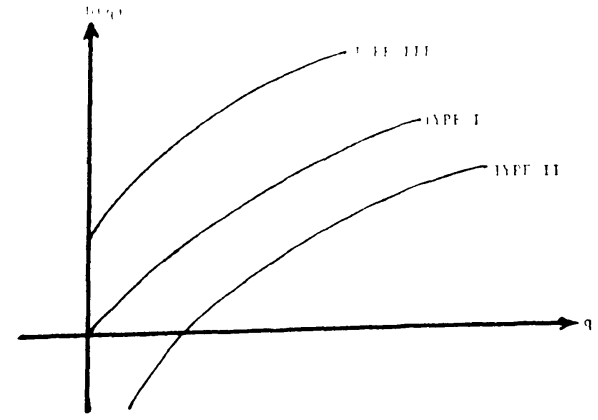

Figure 21

PROPOSITION 2.1. If an optimal solution to the control problem of Section 1 exists for a strictly concave utility function which also satisfies $U(0)=0$, then optimal $T<\infty$ if and only if $\lim _{q \rightarrow 0} U^{\prime}(q)<\infty$

PROOF. We first demonstrate that the condition $U(0)=0$ ensures that optimal $q(T)=0$ regardless of the magnitude of optimal $T$ For $T$ finite (25) implies that $U(q(T))=0$. In view of the condition, it immediately follows that $g(T)=0$ For the case of infinite optimal $T$, assume, if possible that optimal $q(T)>0$ Equation (25) then implies that $U^{\prime}(q(T))<\infty$ whence $e^{-\delta T} U^{\prime}(q(T))=\lambda=0$, which clearly contradicts (2.2) Therefore $q(T)=0$

We now show that if $\lim _{q \rightarrow 0} U^{\prime}(q)=\infty$, then $q(T)=0$ cannot be optimal for any finite $T$. Assume, if possible, that this is the case Consequently $H(q(T))=H(0)=0$ is optimal. Now since $\lim _{q \rightarrow 0} U^{\prime}(q)=\infty$, we can always construct an alternative admissible path with $q(T)>0$ but sufficiently small such that $e^{-\delta T} U^{\prime}(q(T))>\lambda$ In addition, the strict concavity of $U(q)$ ensures that $\left.U(q(T)))>U^{\prime}(q(T))\right) q(T)$, that is, average utility is greater than marginal utility. Combining the two inequalities yields $H(q(T))>H(0)=0$, which is a contradiction for it implies that $H(0)$ is not optimal.

To complete the proof we show next that if $\lim _{q \rightarrow 0} U^{\prime}(q)<\infty, T \Rightarrow \infty$ cannot be optimal. Once again, assume the contrary. Since optimal $q(t)$ declines continuously to zero, there must exist a $t<\infty$, but sufficiently large, such that along the optimal path $q(t)>0$ and $e^{-\delta t} U^{\prime}(q(T))<\lambda$. But this contradicts the necessary condition (2 2)

This confirms the Highfill-McAsey result, highlighting the exact role of the condition $U(0)=0$.

PROPOSITION 2.2. If an optimal solution to the control problem of Section 1 exists for a Type II utility function, then optimal $T<\infty$ with optimal $q(T)=\widehat{q}>0$ where $\widehat{q}$ is the unique $q$ satisfying $U(\widehat{q})=0$

PROOF. Assume if possible that an optimal path exists with $T=\infty$. Denote the optimal extraction rate by $q^{*}(t)$. It follows that $\dot{q}^{*}(t)<0 \forall t>0$ and that $\lim _{t \rightarrow \infty} q^{*}(t)=0$. Now choose $T^{*}$ such that $q^{*}\left(T^{*}\right)=\widehat{q} \quad$ Clearly $T^{*}<\infty \quad$ Consider now an arbitrary $T \in\left(T^{*} \infty\right)$ and definite the extraction path $q(t)=q^{*}(t)+\left(x^{*}(T) / T\right) \forall t \in[0 T]$ where, by definition, $x^{*}(T)=x_{0}-\int_{0}^{T} q^{*}(t) d t$. Clearly, the path is feasible and has the property $U(q(t))>U\left(q^{*}(t)\right) \forall t \in[0 T]$ Since $U\left(q^{*}(t)\right)<0 \forall t \epsilon[T \infty)$, this in turn implies

$$
\int_{0}^{T} U(q(t)) e^{-\delta t} d t>\int_{0}^{T} U(q *(t)) e^{-\delta t} d t+\int_{T}^{\infty} U(q *(t)) e^{-\delta t} d t=\int_{0}^{\infty} U(q *(t)) e^{-\delta t} d t .
$$


Clearly this contradicts that $q^{*}(t)$ is optimal with $T=\infty$ Next, since optimal $T<\infty$, the transversality condition ensures that optimal $q(T)=\widehat{q}$

PROPOSITION 2.3. (i) If an optimal solution to the control problem of Section 1 exists for a Type III utility function, optimal $T$ is always infinite (ii) If $\lim _{q \Rightarrow 0} U^{\prime}(q)<\infty$, however, there exists a $T^{*}<\infty$ such that along the optimal path $x\left(T^{*}\right)=0$

PROOF. (i) Since $U(q(t))>0$ always, unboundedness of the optimal time (planning) horizon is obvious from (25) (ii) Choose $T^{*}$ such that $U^{\prime}(0)=\lambda e^{-\delta T^{*}} \quad$ Since $U^{\prime}(q)<\infty \forall q \geq 0$, such a $T^{*}$ exists and $0<T^{*}<\infty$ Now define the linear transformation $V(q)=U(q)-U(0)$ Clearly $V(q)$ is a non-negative, strictly concave utility function such that $V(0)=0, \infty>V^{\prime}(q)=U^{\prime}(q)>0$, and $V^{\prime \prime}(q)=U^{\prime \prime}(q)<0 \forall q \geq 0 \quad$ Now if we replace $U(q)$ by $V(q)$ in the control problem of Section 1, Proposition 21 ensures that the optimal planning horizon for the new problem is $T^{*}$ and the optimal extraction rate until $T^{*}$ is exactly the same as that of the original problem Clearly then $x\left(T^{*}\right)=0$.

The intuitive explanation of the preceding proposition lies in distinguishing between the extraction and planning horizons While $T$ is the planning horizon, extraction horizon is the period during which a positive amount of the resource is extracted As long as positive additions to the utility stream are possible - as is indeed the case with Type III utility function - there can be no limit to the length of the planning horizon On the other hand, positive extraction may last only as long as the present value of benefits from extraction (which decline continuously) is at least as large as the cost or shadow price, $\lambda$, of extraction If the utility function is such that this is possible only for a finite period of time, then extraction must come to a half after a finite period of time

Before concluding, we must note that Vousden [2] also puts forward similar results But the proofs presented here are considerably different and, we believe, much simpler.

\section{REFERENCES}

[1] HIGHFILL, J. and McASEY, M, An optimal control problem in economics, Int. J. Math. and Math. Sci. 14 (1991), 537-544

[2] VOUSDEN, N., Resource depletion with possible non-convexities in production, in J.D. Pitchford and S J. Turnovsky (ed.), Application of Control Theory to Economics, North Holland (1977), 5779.

[3] CHIANG, A.C., Elements of Dynamic Optimization, McGraw-Hill Inc., 1992. 


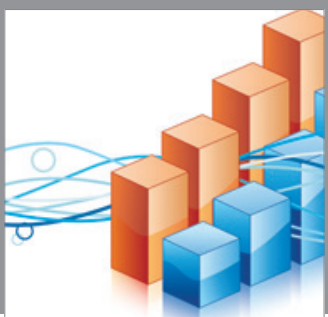

Advances in

Operations Research

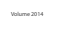

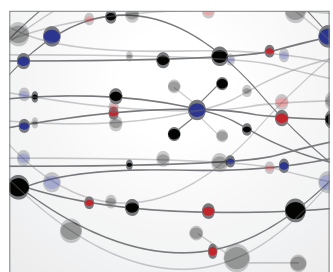

\section{The Scientific} World Journal
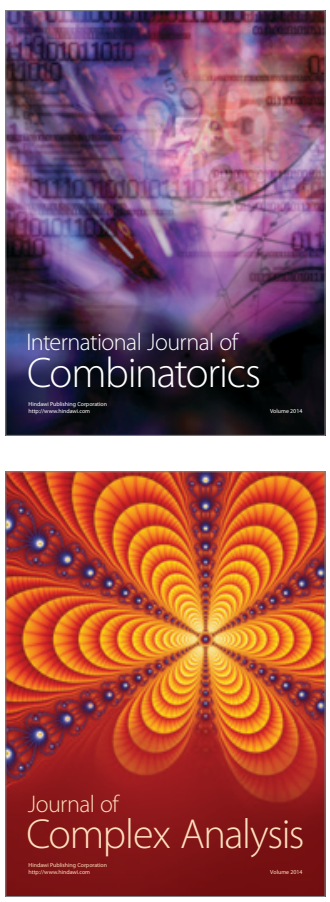

International Journal of

Mathematics and

Mathematical

Sciences
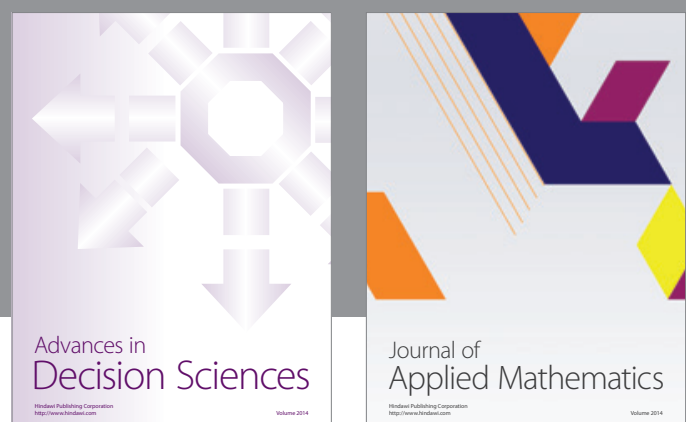

Journal of

Applied Mathematics
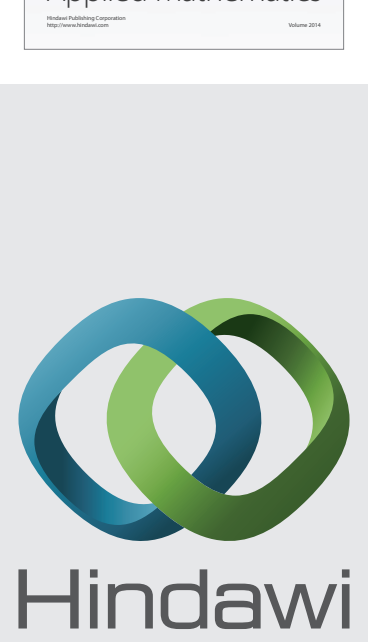

Submit your manuscripts at http://www.hindawi.com
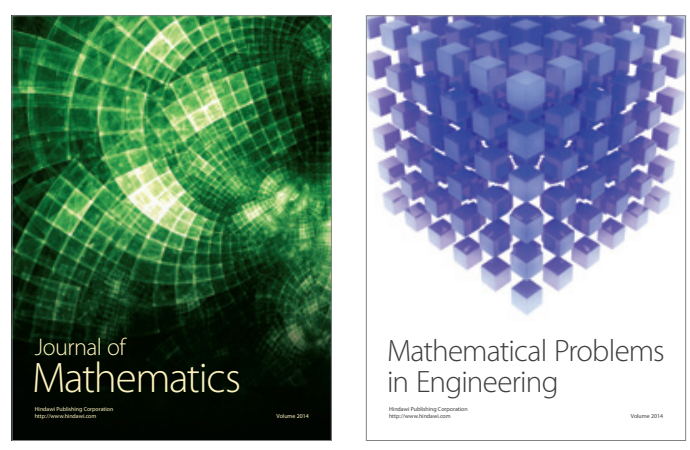

Mathematical Problems in Engineering
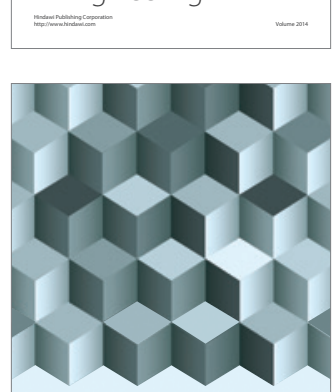

Journal of

Function Spaces
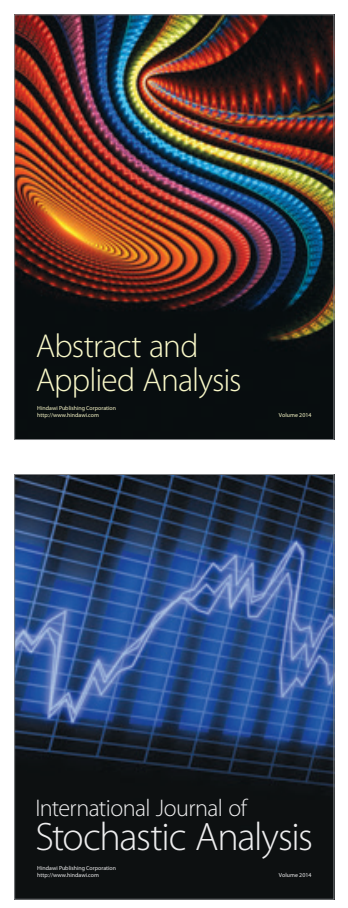

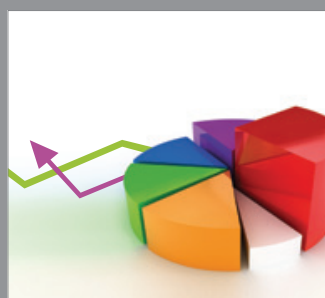

ournal of

Probability and Statistics

Promensencen
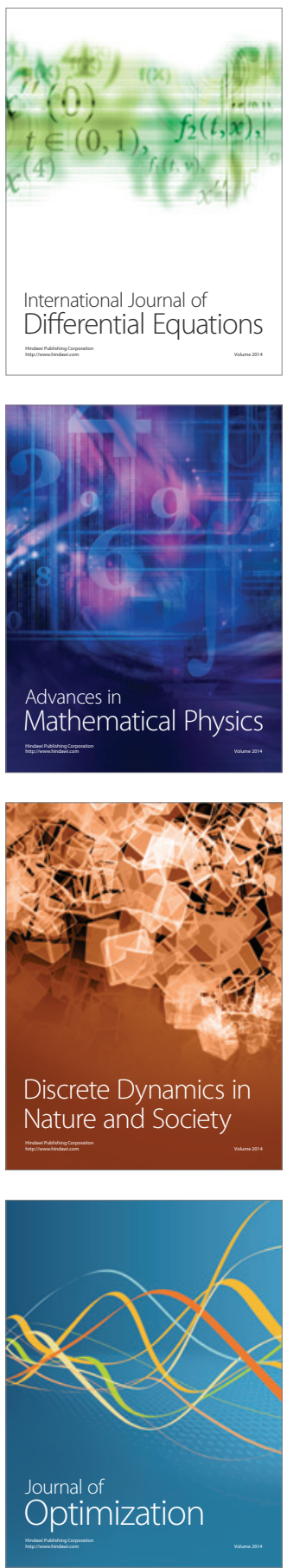